LA COMUNICACIÓN

DE LOS LOBBIES EN

INTERNET

El ciberactivismo de los

\section{Think Tanks}

\section{Antonio Castillo Esparcia}

\section{Profesor}

Facultad de Ciencias de la Comunicación. Universidad de Málaga. Avda. Cervantes, 2, Málaga, 29071 (España) - Email: acastilloe@uma.es

\section{Resumen}

Los Think Tanks son uno de los actores políticos que más desarrollo tienen en la actualidad por su papel sobre la opinión pública. Estos actores políticos desarrollan y proponen acciones concretas sobre el escenario político y uno de sus principales recursos son las estrategias de comunicación en Internet porque les permite llegar a una gran número de ciudadanos de manera directa y fomenta las interacciones entre los Think Tanks y sus públicos.
Palabras clave

Comunicación política, Think Tanks, relaciones

públicas, internet

Key Words

Political communication, Think Tanks, public

relations, internet

\section{Abstract}

The Think Tanks is one of the political actors whom more development they have at the present time by his paper on the public opinion. These political actors develop and propose concrete actions on the political scene and one of their main resources is the strategies of communication in Internet because it

allows them to arrive at a great number of citizens of direct way and foments the interactions between the Think Tanks and its public. 


\section{Introducción}

La comunicación política está imbricada por múltiples actores y numerosas estrategias de comunicación para conseguir los objetivos sociales y políticos. Los partidos políticos han desempeñado un papel de selección de las élites políticas, la preparación de un programa electoral y la lucha por alcanzar el poder político. En ese contexto otras organizaciones sociales no persiguen alcanzar el poder sino participar en el proceso de elaboración y de implementación de políticas públicas. En este contexto surgen los grupos de presión, los lobbies y los Think tanks.

En toda esa dinámica de estructuración simbólica del poder y del ejercicio del poder, la comunicación ha desempeñado un papel esencial como canal de conexión entre las organizaciones y los ciudadanos. Ese papel vertebrador de una opinión pública libre ha conllevado la existencia de múltiples entidades sociales y políticas que muestran una representación social y desarrollan estrategias de comunicación para proponer el conocimiento de sus propuestas y la consiguiente aceptación social y política. Frente a una estructura mediática que se centraba en los medios de comunicación, en nuestros días, los actores políticos planifican, desarrollan y ejecutan acciones en Internet.

Las ventajas de esas acciones son un mejor acercamiento a los ciudadanos con una comunicación de organización a persona (B2P) mediante la personalización de la comunicación y la gestión específica de la comunicación a partir de las necesidades informativas de los ciudadanos. Eso mejora la gestión de los públicos y permite establecer relaciones estables, satisfactorias $y$ permanentes.

\section{Objetivos}

Este artículo persigue conocer el papel de los Think Tanks en el juego político y las acciones específicas de comunicación insertadas en un contexto digital. Como objetivos específicos se pretende establecer un cuerpo teórico sobre estas organizaciones, conocer la dinámica comunicativa, especificar acciones de comunicación y diferenciar su papel respecto de los lobbies. 


\section{Metodología}

Como estudio teórico-práctico de tipo exploratorio, la metodología se concretará en la revisión de literatura sobre el objeto de estudio para estructurar conceptualmente a los Think Tanks y el análisis de las estrategias de comunicación de las princi- pales entidades de América Latina según la calificación establecida por McGann (2007) a través de la aplicación de una plantilla de análisis de contenido a las páginas web de los Think Tanks analizados.

\section{Una aproximación al papel de los Think Tanks}

La difusión del sufragio universal, el surgimiento de los partidos políticos populares dirigidas a movilizar al electorado, la aparición y organización de los think tanks, grupos de presión, que expresan los intereses de los diversos sectores de una sociedad compleja y tienden a influir sobre las decisiones públicas; y el desarrollo de los medios masivos de comunicación nos ha permitido descubrir otras funciones políticas, que no podían ser apreciadas, en su totalidad, en los siglos XVIII y XIX.

El Estado contemporáneo ya no se limita a crear un orden subjetivo para la acción de los particulares, a través del establecimiento de normas jurídicas y a asegurar la vigencia de estas normas, dejando que sea la misma sociedad quién cree sus propias estructuras, sino que actúa mediante diversas políticas -monetaria, educativa, de fomento, fiscal,...- para estructurar, en cierta medida, la coexistencia de los ciudadanos.

En una perspectiva en la que la acción del Estado en la sociedad es remarcable, parece normal que los intereses sociales intentan participar en el proceso político de las decisiones imperativas. La presencia de acciones estatales en determinaciones que afectan a la dinámica interna de la sociedad ha hecho que, desde ésta, los individuos y las asociaciones pretendan intervenir en el proceso creador de unos outputs que incidirán sobre el conjunto de seres sociales. Esta creciente imbricación entre sociedad y Estado fue remarcada por Morgentau:

"La esfera económica ha perdido cualquier autonomía del pasado, ya que ahora está sujeta al control político y trata de controlar las decisiones políticas. Nos encontramos en presencia del revivir de la verdadera economía política (...). Dado que el Estado ejerce un fuerte control, limitación y estímulo de la vida económica, la capacidad para influir en las decisiones económicas del Gobierno se convierte en un elemento indispensable de la competencia para el beneficio económico. La competencia económica se manifiesta inevitablemente en la competencia para la influencia política" 
Se podría decir que se han producido dos fenómenos: una estatalización de la sociedad (mayor presencia del Estado en la sociedad) y una socialización del Estado (la propia sociedad interviene, cada vez más, en las decisiones estatales) ${ }^{i i}$. Esta interilación entre grupos y Estado implica que la actual sociedad no sólo es pluralista, sino que ha de ser pluralista.

\subsection{La comunicación y la construcción de la opinión pública}

En el sistema democrático influir sobre la opinión pública es hacerlo indirectamente sobre el poder político. Todo poder adiciona un grado de dificultad en su actividad cuando las medidas que pretende adoptar cuentan con una opinión pública desfavorable. Conocedores de esta situación, los grupos de presión realizan acciones sobre el público, como un ensayo de persuasión para desarrollar el reclutamiento o con la intención de crear y obtener un clima de simpatía y comprensión general acerca de sus demandas y aspiraciones ${ }^{\mathrm{iii}}$.

Uno de los principales poderes vertebradores entre sociedad y sistema político son los medios de comunicación. Tocqueville (1980: 174-175), en su análisis de la democracia en los Estados Unidos, afirma que los medios de comunicación permiten llegar hasta el más ínfimo lugar de la vida política, iluminando todos los resortes secretos de la política y forzando, de esta manera, a los hombres públicos a comparecer ante la opinión pública.

Los medios de comunicación son para Wright Mills (1978: 315), uno de los mayores instrumentos de poder, cada vez de forma más significativa, de los que disponen las élites de la riqueza y del poder. Esta situación social permite percibir a los mass media como receptores de todo tipo de actividades para poder incidir e influir sobre sus contenidos y temario, enmarcándose en una perspectiva que acrecienta su papel en una sociedad, cada vez, más comunicada cuantitativa y ¿cualitativamente?.

En este sentido, Lester Milbraith (1963: 139) señala que los gobernantes elaboran una decisión a partir de lo que ellos perciben y no de lo que es objetiva u ontológicamente verdadero o real. Por lo tanto, una forma de influir sobre la decisión es incidir sobre las percepciones de los decisores públicos.

El juego político se organiza y estructura sobre la opinión pública en su vertiente moderna y la lucha política tiende a reducirse, cada vez más, a la batalla para conquistar la opinión. Lo esencial de la dominación política reside en el mecanismo fundamental de orden simbólico. La acción política más importante se presenta de manera solapada y subrepticia, al consistir sobre todo en la imposición de sistemas de clasificación del mundo social. 
En este sentido, la irrupción y configuración de los partidos políticos, la generalización del sistema democrático con la celebración de elecciones periódicas, universales y libres se han enmarcado como los factores legitimadores de la soberanía popular (opinión pública). Sin embargo, este concepto se utiliza como un término unívoco y singular que presupone la existencia de una única opinión pública, perspectiva susceptible de criticar ante la existencia diáfana de múltiples opiniones públicas.

El principal problema de esta pluralidad opinativa de los individuos proviene de la limitación discursiva de nuestra sociedad, que postula una jerarquización de las opiniones que circulan en la sociedad. Así, al mismo tiempo que se muestran privilegiados unos determinados discursos, otros se ven arrinconados y silenciados, función facilitada por la posibilidad de disponer de un acceso privilegiado a los medios de comunicación. De esta manera, aquellos sujetos o instancias sociales que desarrollan estrategias discursivas más poderosas pueden presentar una imagen de aquello que desea la opinión pública, cuando en la realidad supone el éxito de una de esas opiniones.

Toda esta intencionalidad mediática está coadyuvada por elementos intrínsecos al sujeto emisor (número de seguidores, tipo de relación con el sistema comunicativo, capacidad financiera,...) y externos (consonancia con la cultura de la sociedad, confluencia con otras opiniones simila- res,...). De la conjunción de estas variables aparece una modalidad y tipología de opinión pública que interacciona con el resto de opiniones sociales y que de esta lucha simbólica emerge una opinión mayoritaria que se autopostula como la opinión pública $y$, por tanto, expresión de todo aquello que desean y pretenden los ciudadanos.

En una complejidad social integrada por numerosos intereses convergentes, pero también divergentes, pensar que existe una única opinión pública supone una cierta dificultad conceptual. A esta realidad podemos añadir que los medios de comunicación son capaces de presentar una imagen de la opinión ficticia, estableciendo y presentando las percepciones, principios $\mathrm{y}$ criterios legitimadores de aquellos que acceden al sistema mediático. Todo este contexto permite afirmar que se produce una circulación constante de imágenes sociales a través del sistema comunicativo.

La presencia de un mercado simbólico que presenta el conjunto de pensamientos sociales es objeto de una lucha para conquistar la mayor posición con la pretensión de imponer "sus productos" (pensamientos, demandas, propuestas,...) y restringir el acceso de los otros.

Así los criterios de los que tienen la capacidad para imponer sus deprecaciones pueden asentar como opinión pública general, la suya parcial y concreta, desvirtuando la legitimación de la opinión pública como representación objetiva y neutra de los pensamientos colectivos. Dentro del con- 
junto de opiniones que coexisten en la sociedad, se manifiestan implícitamente una jerarquía opinativa.

Bajo esta percepción, la creación de ilusiones se concreta en una confusión entre la opinión pública con la presentación pública de la opinión, que remarca e incide sobre unos aspectos, soslayando otros. En este momento, la información general de aspecto informador, general y público deviene en un mensaje comunicativo de contenido persuasivo e imbricado por estrategias comunicativas, con elementos de propaganda y de relaciones públicas. Aspecto resaltado por Núñez Encabo (1996:227) como una dominación de los más poderosos y activos comunicativamente:

"Hasta hace poco tiempo existía en la sociedad una clara diferencia entre los poderes públicos y los poderes económicos privados, estos últimos podían condicionar sólo indirectamente la acción de los poderes públicos. Hoy, por el contrario, los poderes económicos pueden ejercer directamente como poderes públicos sociales a través de la propiedad de los medios o de su financiación, utilizándolos con finalidad política”.

Sin embargo, esta expresividad social no está percibida como una visión atomista de la sociedad, sino que los individuos han sido sustituidos por los grupos de presión como instrumentos canalizadores de las opiniones sociales, opiniones que interaccionan socialmente con los diferentes intereses divergentes y convergentes. Así lo que intentará toda asociación es proponer, al sistema mediático, sus impetraciones, pensamientos, ideologías, ideas como objetivos válidos y adecuados, con la intención que los medios actúen como amplificadores de su teleología, irradiando al mayor número de personas.

El conjunto de estrategias de comunicación por los think tanks pueden agruparse, sucintamente, en los siguientes parámetros:

1. Difuminar sus deprecaciones, propuestas y demandas sobre el conjunto de los ciudadanos (función difusora).

2. Presentar y exhibir ante los poderes públicos una representación de soporte social, a través de las apariciones mediáticas, que permiten reflejar que el grupo dispone de gran presencia social (función de apoyo social).

3. Establecer y presentar los instrumentos instructivos sobre diferentes problemáticas internas y externas (función educativa).

4. Actuar como elementos sensibilizadores de la sociedad sobre las temáticas asociativas, en relación a las percepciones individuales y colectivas (función sensibilizadora).

5. Posibilidad de restringir el acceso de las propuestas de otros grupos, debido a la limitación temática de la agenda-setting ${ }^{\text {iv }}$. De esta manera, la asociación puede limitar la concurrencia de intereses divergentes a los suyos, creando un clima de propuestas monotemáticas, esto es, las propias (función restrictiva). 
6. Mejorar su participación en el diálogo social interasociativo con instancias gubernamentales (función de relaciones públicas).

7. Mantener una actividad constante en los medios de comunicación sobre sus objetivos y temáticas. Se consigue así una especialización temática que permite presentarse como la organización idónea sobre la cita cuestión (función monopolizadora).
8. Todo think tank que mantenga una cierta presencia, de manera positiva, en los medios incrementa su legitimidad ante los poderes públicos, los propios miembros, el resto de ciudadanos y los propios medios comunicativos. Se pretende introducir la siguiente secuencia: legitimación conseguida $\Rightarrow$ propuestas legítimas (función legitimadora).

\section{Concepto de Think Tank}

Definidos como tanques de investigación, centros de investigación, laboratorios de ideas, centro de pensamiento, tanques de ideas son algunos de los términos que intentan definir a los think tanks. Fruto de su ambigüedad terminológica y funcional los Think Tanks han sido conceptualizados desde diferentes perspectivas y bajo esa denominación podemos agrupar a multitud de organizaciones sociales que planifican, realizan y ejecutan investigaciones sobre determinadas temáticas para proponer propuestas de actuación política. Antes de entrar con profundidad en sus funciones es pertinente resaltar una serie de aspectos definidores:

1. son entidades organizadas con una estructura interna, personal permanente y eventual. Por tanto, huyen de la temporalidad y de las acciones episódicas ya que mantienen una intención de per- manencia. Esa organización está muy relacionada con el acto inicial de creación ya que éste define su estructura. Así, nos encontramos con Think Tanks que surgen por la iniciativa de personas individuales, por un grupo de expertos en una determinada materia, través de una fundación, por iniciativa de profesionales (empresarios, por ejemplo), por formaciones políticas o mediante iniciativa universitaria

2. sin ánimo de lucro, por lo que entre sus objetivos no está el beneficio material e inmediato, sino que buscan el conocimiento y el progreso con relación a un ámbito social (derechos humanos, por ejemplo), un sector de actividad (economía o jurídico), el contexto internacional (relaciones internacionales) o una ideología (liberalismo, entre otros). En este sentido, las temáticas finalistas son 
amplias y extensas. Esa pretensión no lucrativa no impide la necesidad de buscar y conseguir financiación (fundraising) para poder desarrollar sus cometidos y esos recursos están condicionados por la estructura:

si la génesis es una fundación o mediante una aportación singular no existen muchos problemas para buscar estipendios. Eso no invalida la dificultad intrínseca de financiación de cualquier entidad.

- Si surge a partir de la iniciativa de sus creadores se hace innegable la búsqueda de fuentes de financiación y en este aspecto son extensas y complejas. La financiación puede ser ajena mediante aportaciones de otras entidades semejantes (Think Tanks de otros países o fundaciones extranjeras), gubernamentales, socios o donantes con personalidad jurídica (empresas), o donantes individuales. Sobre los recursos propios nos encontramos esencialmente dos vías: venta de productos elaborados o investigación bajo demanda.

Acción comunicativa con pretensión de dar a conocer sus investigaciones y que otras entidades públicas y privadas tengan presente los estudios qie realiza en su ámbito. De ahí que los documentos e informes sean uno de los principales instrumentos de estas organizaciones. Esa capacidad de comunicación se extiende también a la realización de talleres, seminarios o workshops con una pretensión de comunicación directa con las personas interesadas. Sin embargo, uno de los principales ámbitos en los que trabajan es el acceso a los medios de comunicación ya que estos posibilitan llegar a numerosos individuos y confieren presencia e influencia social. Igualmente, en la actualidad el papel que desempeña Internet ha exigido que se estructuren acciones específicas de comunicación en la red y de esa manera llegar más directamente (sin intermediarios, los medios de comunicación) a los públicos implicados y sensibilizados.

"Todavía no es comparable a la televisión como difusor de mensajes y propaganda política, pero Internet va afianzando su posición y un estudio del Partido Republicano afirma que el $37 \%$ de los adultos estadounidenses utiliza la Red para informarse sobre política. En este contexto, los blogs despuntan como creadores de opinión. Ésta es la principal conclusión de la segunda edición del Foro de Democracia Personal (PDF, en sus siglas en inglés), celebrado esta semana en Nueva York, en el que han participado los principales gurús de la política en Internet" (Maria Silva, 'Blogs, los 'think tanks' del S. XXI”, en Diario El País, 18 de mayo de 2005)

- En este sentido, tal como se ha señalado anteriormente los think tanks con sus estrategias de comunicación persiguen la difusión de su pensamiento, la legitimidad para proponer propuestas y la monopolización de una temática como 
entidad especializada e interlocutora (legitimada) en ese ámbito para los organismos y personas públicas.

- Acción política referida a que poseen un objetivo específico para influir en las decisiones políticas a través de las propuestas específicas sobre implementación de políticas públicas. Los think tanks poseen un marcado carácter diferenciador y presencial ya que investigan y elaboran propuestas concretas de actuación. Esta pretensión ha mostrado la existencia de think tanks que se alejan un poco de su objetivo autónomo e inicial del poder político ya que nos encontramos con think tanks que son creados y financiados, en parto o su totalidad, por partidos políticos y son órganos en los que se elaboran propuestas específicas de la oferta programática de los partidos políticos. En este aspecto, sí que cuadra perfectamente la expresión laboratorio de ideas puesto que, una de sus principales funciones, es la de elaborar propuestas que se incluyen en los programas partidistas.

Atendiendo a estos requisitos formativos de los think tanks se pueden definir como entidades que a través de la investigación y el análisis, proponen propuestas de actuación política a los órganos institucionales mediante estrategias de comunicación directa o indirectas, como es el acceso e influencia sobre la opinión pública. Con este concepto, considero que se amplía una definición inicial en la que define a los think tanks como organizaciones formadas por intelectuales y analistas diversos que reciben contratos o encargos (de organizaciones públicas y privadas) para analizar ideas y proyectos y proponer formas de aplicación de diferentes actividades gubernamentales $\mathrm{y} / \mathrm{o}$ industriales. Finalmente cabe señalar que tres son los elementos que contextualizan la actividad de un think tanks:

- Investigación para generar ideas y propuestas

- Proposiciones concretas, detalladas y reales hacia el poder público

- Generación de estrategias de comunicación para la opinión pública y los órganos institucionales. Con ello va implícito el término esencial como es la influencia sobre los intelectuales, políticos, empresarios, ciudadanos, organizaciones, etc.

El estudio realizado por McGann define a los, think tanks como organizaciones de investigación, análisis e implementación de políticas públicas que generan investigaciones, análisis y recomendaciones en temas nacionales e internacionales que facilitan a los actores políticos y a la sociedad en general tomar decisiones de manera informada sobre temas de políticas pública.

Stone define a los think tanks como "institutos de investigación independiente cuyo principal objetivo es la investigación de las políticas públicas, [...] organizaciones no gubernamentales sin fines de lucro, inde- 
pendientes del gobierno, partidos políticos y grupo de interés" (1996: 16).

Abelson los define como "institutos orientados a la investigación, sin fines de lucro y no partidarios (que no significa que no sean ideológicos) cuyo principal objetivo es influenciar en la opinión pública y en las políticas públicas” (2002)

\subsection{Herramientas de comunicación}

La irrupción de las tecnologías de la comunicación ha modificado la modalidad y tipología de la comunicación de las organizaciones. Frente a una etapa anterior en la que las relaciones entre organizaciones y públicos estaba mediatizada por el acceso a los medios de comunicación, en la actualidad las salas de prensa virtuales, los blogs, las páginas web, la comunicación viral o los podcasts permiten una mayor relación directa con los públicos de las organizaciones.

Las nuevas formas de comunicación que están surgimiento debido a la extensión de las tecnologías posibilitan que las organizaciones (empresas, Organizaciones no Gubernamentales, asociaciones sociales, sindicatos, partidos políticos,...) pueden establecer relaciones directas con los públicos que les afectan (relaciones públicas). Estos públicos implicados disponen de canales directos con las organizaciones lo que coadyuva a que se alcance uno de los principales principios teóricos de la comunicación en las organizaciones como son las estrategias en tiempo real y el acceso directo a los públicos (stakeholders).

La comunicación en las organizaciones se viene desarrollando a partir de las estrategias que se generan desde las organizaciones y que se dirigen a los públicos, adoptando éstos papeles más de pasividad que de actividad. Sin embargo, la aplicación de tecnologías permite facilitar las comunicaciones de los públicos con las organizaciones (correo electrónico, chats, enlaces con páginas webs, etc.) y disponer de las demandas societales de manera más sencilla y rápida. Asimismo, las organizaciones pueden evaluar la incidencia de sus estrategias y el grado de decodificación e interiorización por parte de los receptores.

En este sentido, Almansa (2008: 5) ha señalado el papel de las tecnologías en el proceso de interacción e intercambio entre organizaciones y públicos, como una manera de acercar las demandas sociales a las organizaciones. Desde esta perspectiva, los Think Tanks recurren a Internet para crear estados de opinión y de comprensión sobre temáticas públicas.

La irrupción de tecnologías ha favorecido los flujos de comunicación interpersonales e interorganizativos modificando estrategias, objetivos y herramientas. Las sociedades complejas y la expansión de Internet han supuesto una creciente capacidad de las relaciones públicas de expandir su actividad, por un lado, y de especializar las estrategias segmentando públicos, por otro lado. En este sentido, las relaciones con los 
medios de comunicación también se han visto afectadas por esos nuevos avatares y surgen modalidades de interacción entre organizaciones y medios. Una de estas mutaciones han sido las denominadas Salas de Prensa Virtuales definidas como espacios comunicativos en red que contienen las herramientas y las actividades dirigidas a los medios de comunicación por parte de las organizaciones

\subsubsection{Estudio sobre las herramientas}

El principal objetivo que presentan los Think Tanks es su capacidad difusora de las ideas que defienden. Son instancias que realizan investigaciones por motivación propia y a instancia de parte pero todas sus estudios poseen un marcado carácter difusor. Esa acción comunicativa les permiten difundir sus estudios y sus propuestas al conjunto de la colectividad ya determinados sectores muy específicos.

En este sentido, deben desarrollar la capacidad de generas documentos de trabajo que se inserten en la sociedad y que se integren en el ecosistema de las ideas sociales. Incluso uno de los iniciadores de estas organizaciones en sus ideas primigenias ya señalaba para difundir las ideas no se debía recurrir a la creación de un partido político sino a centros de estudios que difundieran esas ideas. Para Mato los estadios iniciales del concepto de think tanks tienen lugar a finales de la II Guerra Mundial y como respuesta a la reformulación del pensa- miento liberal que pudiera luchar ideológicamente con los postulados y la expansión del comunismo. Así, el inicio de esa reformulación liberal tiene lugar con la publicación del libro Camino a la servidumbre del profesor Friederich Hayed. Tras esa publicación que se definía como un libro político, un empresario, Anthony Fisher, le confesó a Hayed su intención de crear un partido política ante lo que éste le comunicó que lo mejor era crear un instituto de estudios políticos que renovará las ideas de investigadores, profesores, periodistas, políticos, mediante la realización de productos comunicativos como son publicaciones, conferencias, seminarios que influyeran sobre la opinión pública y ésta sobre los políticos (2005, 139-142).

Para cumplir uno de los objetivos de este artículo se van a analizar una serie de Think Tanks de América Latina según la clasificación establecida el estudio deMcGann (2007) a través del análisis de sus páginas web y de las herramientas de comunicación que utilizan en la difusión de sus investigaciones.

Por tanto, uno de los principales objetivos de los Think Tanks que nos encontramos es la necesidad de informar de las investigaciones y en la educación de la ciudadanía sobre las temáticas que son objeto de estudio. Todas las entidades analizadas poseen páginas web accesibles y con gran cantidad de material disponible para su descarga. Así las herramientas más usuales son: 
- Un web site que sirve de punto de difusión de las investigaciones para que estén a disposición de los interesados. La Corporación Excelencia de la Justicia señala que en 2007 visitaron la web 24.682 personas con una mayoría desde estados Unidos. O el caso de Cadal que apunta a un millón de visitas y que las informaciones más consultadas fueron artículos $(47,27 \%)$, prensa $(14,61 \%)$, entrevistas (6,52\%), documentos $(5,17 \%)$, eventos $(4,7 \%)$, informes $(2,71 \%)$ y libros $(1,64 \%)$

- Las descargas de documentos e informes

- Posibilidad de consultar textos completos de libros publicados por la entidad y suministrados en formato pdf

- Las apariciones en los medios de comunicación ya sea con una reseña en los distintos medios en los que se ha tenido presencia o la posibilidad de conocer cómo se ha reflejado la información en los medios. Así Fiel permite descargar 18 artículos del año 2008

- Incidencia en los medios desde una perspectiva numérica. Cippec señala que durante el primer semestre de 2008, la entidad ha tenido 776 menciones en la prensa

- Vídeos para que puedan ser consultados por los visitantes. Cadal posee 18 vídeos diferentes

- Servir como fuente de información como expertos para los medios. Cippec señala que los principales diarios, los programas televisivos más importantes y los corresponsales extranjeros de los medios más conocidos del mundo convocaron a miembros de Cippec para hablar de los temas clave de 2007

- Conferencias

- Seminarios genéricos o específicos. De presentación de resultados o de capacitación

- Encuentros con expertos nacionales o internacionales

- Simposios y mesas redondas

- Intervenciones en actos académicos

- Publicaciones de libros

- Recepción de personalidades internacionales como Jefes de Estados, miembros de Gobiernos o especialistas académicos

- Boletín electrónico sobre la temática que se investiga

- Posgrados para estudiantes

- Cursos de preparación gratuitos como es el caso de Fiel que organiza un curso gratuito para periodistas sobre economía

- Contacto con legisladores, autoridades del gobierno, dirigentes políticos o empresarios

- Informes temáticos

- Anuarios 


\section{Conclusiones}

La estrategia comunicativa de los Think Tanks se dirige a las propias organizaciones políticas (partidos políticos), a las instituciones (gobierno, legislativo y administración, principalmente) y a los ciudadanos. Respecto de los primeros funcionan mediante encargos específicos o por la especial significación entre Think Tanks y partidos político ya sea porque aquél se crea para generar masa crítica opinativa o por su un relación ideológica (caso de Faes en el Partido Popular o Ideas en el PSOE). Sobre las instituciones, los Think Tanks presentan propuestas concretas de acción pública y se vehiculan mediante contratos específicos de asesoría. También se presentan como instancias de reclutamiento de las élites políticas. Finalmente, sobre los ciudadanos recurren a la elaboración de documentos, las reuniones con especialistas y con públicos especializados, a las referencias informativas en los medios de comunicación y en el establecimiento de relaciones específicas en internet.

\section{Referencias}

ALMANSA, A. (2008): Estudio sobre el uso de las nuevas tecnologías en comunicación institucional, en Revista Icono 14, $\mathrm{n}^{\circ} 11: 1-14$

ABELSON, Donald (2002). Do Think Tanks matter? Assesing the impact of Public Policy Institutes, Montreal: McGill-Queen's University Press

BRYANT, J. y Dolf ZILLMAN (comp.) (1996): Los efectos de los medios de comunicación. Investigaciones y teorías, Barcelona, Ed. Paidós Ibèrica.

MATO, Daniel 2005: Redes de 'think tanks', fundaciones privadas, empresarios, dirigentes politicos, economistas, periodistas y otros profesionales en la promoción de ideas (neo)liberales a escala mundial en Mato, Daniel (coord.) Políticas de economía, ambiente y sociedad en tiempos de globalización (Caracas:

Universidad Central de Venezuela): 131-153

MCGANN, J. (2007): The Global “Go-to Think Tanks. The Leading Public Policy Research Organizations in the World, Philadelphia: Foreign Policy Research Institute
MEYNAUD, Jean (1966): Les groupes patronaux et l'opinion, en Il Politico. Rivista di Scienze Politiche, Anno XXXI n'3: 413-437

MILBRATH, Lester (1963): The Washington Lobbyist, Chicago, Rand McNally

NÚÑ̃Z ENCABO, Manuel (1996): La ambivalencia de los medios de comunicación. Poderes y contrapoderes, en José Fèlix Tezanos (ed.): La democracia post-liberal, Ed. Sistema, Madrid: 215-236

STONE, Diane (1996). Capturing the political imagination. Think Tanks and the Policy Process, London: Frank Cass \& CO. LTD

TOCQUEVILLE, Alexis de (1980): La democracia en América, Alianza Editorial, Madrid

WRIGHT MILLS, C. (1978): La élite del poder, Fondo de Cultura Económica, México 


\section{NOTAS}

${ }^{i}$ H.J. Morgentau: “The New Feudalism” en H.S.Kariel: The Political Ordre, New York, 1970: 321

${ }^{i i}$ En relación a las acciones que los grupos sociales pueden realizar sobre el Estado, con la intención de participar, cada vez más, en una organización que incide profuosamente en su actividad privada, Werner Weber la describe así:

"Si el individuo ya no puede contar consigo mismo para organizar la propia existencia, sino que para dar una base de seguridad a su vida, se ve obligado a insertarse en alguna organización colectiva. Y si los hombres en su existencia de grupo como trabajadores sociales dependen, al mismo tiempo, de las manipulaciones de los mecanismos del Estado, es lógico que aquellos reclamen al organigrama directivo de su grupo que realice presiones, en un sentido favorable al grupo, sobre las citadas manipulaciones". Citado por Jürgen Habermas: Capital monopolista y sociedad autoritaria, Barcelona, 1973: 33).

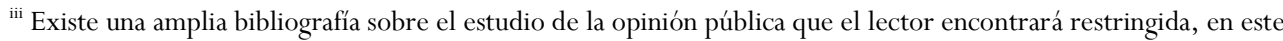
estudio, a aquellas investigaciones que han tenido una repercusión remarcable. Meynaud (1966: 413) cita ejemplos de acción sobre la opinión pública como son los industriales canadienses que en 1866, para incitar una subida de la protección aduanera, crearon una asociación, entre otras cosas, para "estallar el espíritu público" y la Canadian Manufacturers' Association que en 1902 creó un Educational Campaign Committee para publicitar sus objetivos.

iv Sobre las luchas suscitadas entre los elementos que participan en la elaboración de la agenda-setting (medios de comunicación, grupos de presión, partidos políticos, instituciones públicas), véase el artículo de J. McLeod, G. Kosicki y D. McLeod "Expansión de los efectos de la comunicación política”, en Bryant y Zillman (Comp.) 1996: $169-222$. 